\title{
Advanced Lymphoma
}

National Cancer Institute

\section{Source}

National Cancer Institute. Advanced Lymphoma. NCI Thesaurus. Code C153172.

Lymphoma that has spread extensively to other anatomic sites or is no longer responding to treatment. 